\title{
Subaru Coronagraphic Search for Companion Brown Dwarfs
}

\author{
Yoichi Itoh ${ }^{1}$, Motohide Tamura ${ }^{2}$, Saeko S. Hayashi ${ }^{2}$, Yumiko Oasa ${ }^{3}$, \\ Misato Fukagawa $^{4}$, Hiroshi Suto ${ }^{2}$, Koji Murakawa ${ }^{2}$, Takahiro Naoi ${ }^{4}$ \\ ${ }^{1}$ Graduate School of Science $\&$ Technology, Kobe University \\ 1-1 Rokkodai, Nada, Kobe, Hyogo 657-8501, Japan, yitoh@kobe-u.ac.jp \\ 2 National Astronomical Observatory of Japan \\ ${ }^{3}$ National Space Development Agency of Japan \\ ${ }^{4}$ University of Tokyo
}

\begin{abstract}
Near-infrared coronagraphic images of GG Tau and GK Tau are presented as preliminary results of the search for young brown dwarfs around $\mathrm{T}$ Tauri stars.
\end{abstract}

\section{Introduction}

We are now conducting a near-infrared coronagraphic search for young brown dwarfs around $\mathrm{T}$ Tauri stars in the Taurus molecular cloud. The reasons why the study is aimed at such objects are as follows. 1) Brown dwarfs are orders of magnitudes brighter in the young stage than in the mature stage. In a binary system, the magnitude difference is smaller in the young stage than in the mature stage; 2) The binary frequency of objects associated with the Taurus molecular cloud is greater than those of the main-sequence stars by at least a factor of 2 .

However, young brown dwarfs are thought to be still very faint, therefore we need to conduct observations with a large aperture telescope. Moreover, young brown dwarfs as companions are thought to be in close vicinity of the bright central object, therefore we need observations with a high dynamic range as well as high spatial resolution. Coronagraph Imager with Adaptive Optics (CIAO) on the Subaru Telescope is one of the most powerful instruments for the search of such companion brown dwarfs.

\section{Circumbinary Disk around GG Tau}

$H$-band coronagraphic observations of GG Tau were carried out on 2001, January 16. The occulting mask we used was 0.8 in diameter. The $H$-band coronagraphic image of GG Tau is shown in Figure 1, where the circumbinary disk is clearly detected with high spatial resolution and with high signal-to-noise ratio. This image demonstrates the capability of CIAO to detect faint objects in close vicinity of the bright central objects. Details of the observations and results will be presented elsewhere (Itoh in preparation). 

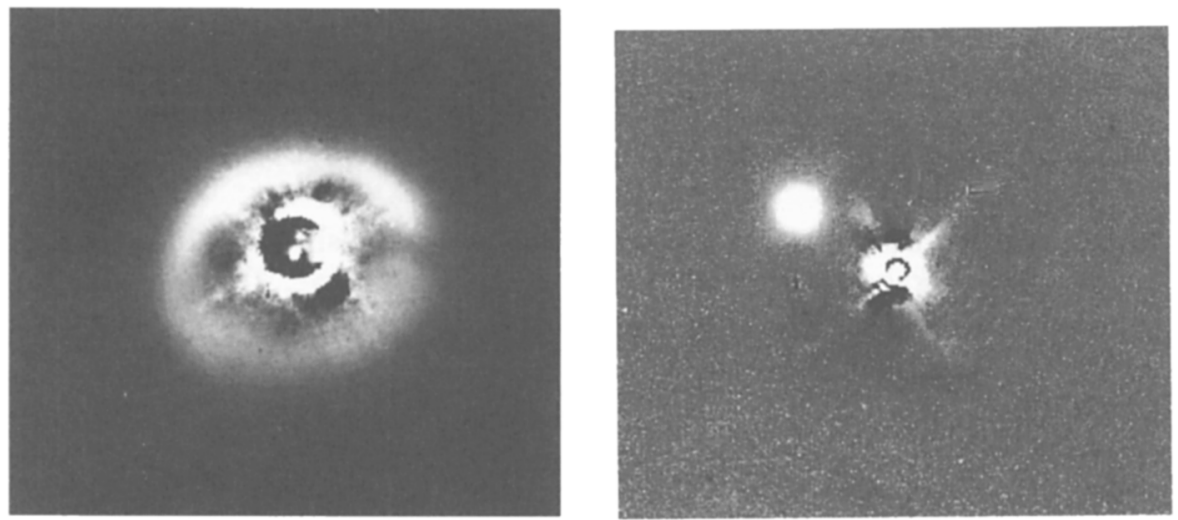

Figure 1. (left) $H$-band coronagraphic image of GG Tau. The PSFs of the GG Tau binary were subtracted. The field of view is $10^{\prime}{ }^{\prime} 16 \times 99^{\prime} \cdot 79$. (right) $H$-band coronagraphic image of GK Tau. The PSF of the GK Tau primary was subtracted. The field of view is $11^{\prime \prime} \cdot 34 \times 10^{\prime \prime} 09$.

\section{Brown Dwarf Candidates nearby GK Tau}

GK Tau is a wide binary system accompanied by GI Tau with a separation of $13^{\prime \prime}$. The age of GK Tau is estimated to be about $1 \mathrm{Myr}$, based on the evolutionary track of Baraffe et al. (1998). $H$-band coronagraphic observations were carried out on 2001, December 6. The mask we used was $0{ }^{\prime} 5$ in diameter. The $H$-band coronagraphic image of GK Tau is presented in Figure 1. A companion is clearly detected with a separation of 2 ! 55 (360 AU). This companion was previously detected by $z$-band direct imaging observations (Reipurth \& Zinnecker 1993), whereas no detection was accomplished by near-infrared observations. This is because the previous near-infrared searches were conducted by direct imaging, speckle, and lunar occultation, all of which did not have enough flux sensitivity and/or dynamic range. The $H$-band magnitude of the companion is derived to be 11.8. If the age and visual extinction of the companion are the same as those of the primary, the mass of the companion is estimated to be $0.05 \mathrm{M}_{\odot}$.

Signal-to-noise ratio of the GK Tau companion is more than 100. According to the evolutionary track of Baraffe et al. (1998), a $0.02 \mathrm{M}_{\odot}$ brown dwarf with an age of $1 \mathrm{Myr}$ in the Taurus molecular cloud has an $H$-band magnitude of 13.9. Therefore we expect a few minutes integration will be able to detect young brown dwarfs with $\mathrm{S} / \mathrm{N}=10$.

\section{References}

Baraffe, I., Chabrier, G., Allard, F., \& Hauschildt, P. 1998, A\&A, 337, 403 Reipurth, B. \& Zinnecker, H. 1993, A\&A, 278, 81 